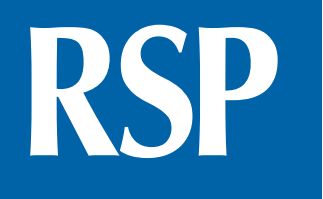

http://www.rsp.fsp.usp.br/
Revista de Saúde Pública

\title{
Prevalence and factors associated with active transportation to school for adolescents
}

\author{
Edina Maria de Camargo' (iD, Michael Pereira da Silva" iD, Jorge Mota"l' iD, Wagner de Campos ${ }^{\mathrm{IV}}$ \\ I Universidade Federal do Paraná. Programa de Pós-Graduação em Educação Física. Curitiba, PR, Brasil \\ " Universidade Federal do Rio Grande. Faculdade de Medicina. Rio Grande, RS, Brasil \\ III Universidade do Porto. Faculdade de Desporto da Universidade do Porto. Departamento de Educação Física. \\ Porto, Portugal \\ iv Universidade Federal do Paraná. Departamento de Educação Física. Curitiba, PR, Brasil
}

\section{ABSTRACT}

OBJECTIVE: To verify the prevalence and factors associated with active transportation to school (ATS) among Brazilian adolescents attending public schools.

METHODS: Crossectional study with a representative sample of 1,984 adolescents (55.9\% girls). Sociodemographic variables included were: gender, age, parental schooling, and socioeconomic status. Psychosocial factors included were: social support from parents and friends for physical activity. Walking, cycling, or skateboarding to school were considered models of active transportation. Binary logistic regression models verified sociodemographic and psychosocial factors association with ATS, adopting $\mathrm{p}<0.05$.

RESULTS: The prevalence of active transportation to school was 37.7\% (16.2\% boys and $21.5 \%$ girls). For boys, ATS was associated with: social support from parents in practicing physical activity together as a family $(\mathrm{OR}=1.57 ; 95 \% \mathrm{CI} 1.09-2.25)$, giving them rides $(\mathrm{OR}=1.56$; $95 \% \mathrm{CI}$ 1.04-2.32), and remarking their good performance on it ( $\mathrm{OR}=1.73$; CI95 1.08-2.76); as well as the social support from friends in practicing physical activity together ( $\mathrm{OR}=2.23$; 95\% CI 1.35-3.69). For girls, the likelihood of using ATS increased with age (OR $=1.43$; 95\% CI 1.06-1.92) and having friends who practice physical activity together with them $(\mathrm{OR}=1.48,95 \% \mathrm{CI} 1.04-2.10)$.

Edina Maria de Camargo

Rua Coração de Maria, 92

Campus Jardim Botânico

80210-132 Curitiba, PR, Brasil

E-mail: edinacamargo@gmail.com

Received: Sep 12, 2019

Approved: Nov 25, 2019

How to cite: Camargo EM, Silva MP, Mota J, Campos W. Prevalence and factors associated with

active transportation to school for adolescents. Rev Saude Publica.

2020;54:78

Copyright: This is an open-access article distributed under the terms of the Creative Commons Attribution License, which permits unrestricted use, distribution, and reproduction in any medium, provided that the original author and source are credited.

CONCLUSION: Age and social support for physical activity were associated with ATS. Parents who practice together, give rides, and remark on physical activities increase the likelihood of adolescent boys using ATS. Social support from friends to physical activity increased the likelihood of both genders using ATS.

DESCRIPTORS: Adolescent. Walking. Motor Activity. Socioeconomic Factors. Cross-Sectional Studies. 


\section{INTRODUCTION}

Active transportation to school (ATS) is an effective approach to improve overall physical activity (PA) levels among adolescents ${ }^{1}$. Adolescents involved in ATS, such as walking and cycling, accumulate more PA and have lower cardiometabolic risks such as overweight and obesity, diabetes, and metabolic syndrome ${ }^{2-4}$. Despite the potential health benefits of ATS, studies have reported a drop in the proportion of children and adolescents using it in recent decades ${ }^{2-4}$, which may contribute to a global decline in PA levels. Thus, promoting ATS has featured in international initiatives aimed at increasing PA at the population level ${ }^{3-6}$.

Ecological models state that health behaviors, such as active transportation, are influenced by various factors at multiple levels, including psychosocial and environmental factors ${ }^{5,6}$. Such factors may vary according to gender, age, parental schooling, and socioeconomic status $^{5,6}$. As an example, adolescents living near school are more likely to use ATS ${ }^{1,2}$; however, older adolescents often use passive transportation, such as car and motorcycle, even to nearby destination $\mathrm{s}^{1,2}$. These results suggest a need for greater efforts to understand factors that may influence the use of ATS, aiming to increase the use of this mode of commuting to school and other destinations ${ }^{1,2}$.

In recent years, Brazil have attempted to improve the urban environment by creating bike paths and bike lanes, which may favor the use of active transport modes by the population. ${ }^{7}$ This type of initiative have a medium- to long-term impact, as well as a wide reach ${ }^{8}$. Understanding the relation between sociodemographic and psychosocial factors - specifically the support from parents and friends to practice physical activity in different contexts (leisure and transportation) - may provide valuable information to public health authorities in implementing and promoting ATS and, consequently, improving PA levels. This study aimed to investigate the prevalence and factors associated with active transportation to school among Brazilian adolescents attending public schools.

\section{METHODS}

This is a cross-sectional study conducted in 2018, with a representative sample of adolescents aged from 15 to 17 years, attending public high schools in Curitiba, in the state of Paraná, Brazil. This study followed the recommendations of the National Health Council (Resolution No. 466/2012) for research involving human beings, and it was approved by the Research Ethics Committee of the Universidade Federal do Paraná (CAAE: 98133218.8.0000.0102) and authorized by adolescents' parents or legal guardians upon signing the informed consent form.

According to the 2017 school census, conducted by the National Institute for Educational Studies and Research Anísio Teixeira (INEP), the public schools of the city had 53,760 adolescents, of both genders and aged from 15 to 17 years, enrolled in high school. $\mathrm{G}^{*}$ Power was used to estimate the sample. We adopted a 1.49 prevalence ratio (PR) between social support and physical activity ${ }^{9}, 50 \%$ prevalence of active transport ${ }^{2}, 95 \%$ confidence level $(\alpha=0.05)$ with $80 \%$ power $(\beta=0.20)$, and a $30 \%$ increase to compensate possible losses and refusals. Estimated necessary sample size was 1,930 adolescents: 965 boys and 965 girls.

Sampling procedure was initiated by conglomerates, in three stages. First, all public schools within each of the nine administrative regions of Curitiba were stratified; second, two schools of each region were drawn; third, one class of each high school year was randomly selected, considering the number of students required by gender for a given administrative region of the municipality. The random selection contemplated both morning and afternoon classes. All students from the selected classes were invited to participate in the study. 
The total of 2,506 adolescents were invited to participate in the study. Those who failed in presenting the informed consent form signed by a parent or legal guardian ( $\mathrm{n}=$ 100), those who refused to participate in the study, and those who were absent on the collection day $(\mathrm{n}=56)$ were not included. We excluded those who reported physical or cognitive limitations associated with PA practice $(\mathrm{n}=12)$ and those aged 18 years $(\mathrm{n}=$ 125). Adolescents who answered the questionnaires incorrectly $(n=229)$ were considered sample loss. 1,984 adolescents composed the study analytical sample. A posteriori power analyses showed that this sample could identify statistically significant prevalence ratios above $\mathrm{OR}=1.28$ as an increase in the use of active transportation to school, and below $\mathrm{OR}=0.77$ as lower likelihood in using active transportation to school, considering a $34 \%$ prevalence of adolescents with poor social support and without the habit of using active transportation.

\section{Gender, Age, Schooling, and Socioeconomic Status}

Gender was self-reported (male or female) and age was estimated from the date of birth informed by the adolescent and classified into 15, 16, or 17 years. Parental schooling, as well as socioeconomic status, were classified according to the Brazilian Association of Research Companies (BARC) ${ }^{10}$, into the following categories: elementary school, high school, or college (we asked for father and mother's schooling and the head of the household). The Brazilian Criteria of Economic Classification (BCEC) $)^{10}$ classifies the social strata into the economic classes A, B1, B2, C1, C2, and D-E, based on data from the National Household Sample Survey (NHSS). For analysis, and aiming to ensure comparability to related studies, socioeconomic status (SES) were classified into three categories: lower (classes $\mathrm{C}+\mathrm{D}+\mathrm{E}$ ), middle (B1+B2), and higher (A1+A2).

\section{Social Support from Parents and Friends}

Social support from parents and friends for PA practice was measured using a 10-item scale - the ASAFA Scale, - which presents satisfactory internal consistency (parents: $\alpha \geq 0.77$ and composite reliability index [CRI] $\geq 0.83$; friends: $\alpha \geq 0.87$ and $\mathrm{CRI} \geq 0.91)^{11}$. The adolescents reported the frequency (never $=1$, rarely $=2$, often $=3$, always $=4$ ) with which parents and friends offered some kind of social support for PA practice (encourage, practice, ride, assist, remark, invite) during a typical week ${ }^{11}$, by answering the questions: "How often do your parents: Encourage you to practice PA? Practice PA with you? Give you a ride or provide transportation for you to go to the place where you practice PA? Watch you practicing PA? Remark your good performance in the PA?" and "How often do your friends: Encourage you to practice PA? Practice PA with you? Invite you to practice PA with them? Watch you practicing PA? Remark your good performance in the PA?"

For analysis, and aiming to ensure comparability to related studies ${ }^{12,13}$, "rarely" and "frequently" were grouped and classified as "sometimes." Weekly frequency of PA was classified as never, sometimes or always.

\section{Active transportation to school}

We assessed the used mode of transportation to and from school during a typical week (walking, biking, skateboarding, bus, van, or car). Students who reported walking, cycling, or skateboarding to and from school were considered "active"; the others were considered "passive." This question presented adequate test-retest reliability (intraclass correlation coefficient $0.90-0.95 ; \mathrm{p}<0.05$ ) and has been applied by related studies ${ }^{14,15}$.

\section{Data Analysis}

To avoid bias related to the complex sampling process (stratified cluster), association analyses were corrected by the complex delineation, using the complex sample command in the SPSS Statics 23.0. Such procedure was adopted to ensure that estimates would reflect population data from the elementary sampling units. 
Prevalence was described by relative and absolute frequency distribution. Pearson's chi-square test was used to compare proportions between genders; then a post hoc test was used to demonstrate which categories showed the greatest difference. The crude and adjusted binary logistic regression was applied to verify the association of demographic factors and social support from parents and friends for ATS. Following the stepwise criterion, $p$-value $\leq 0.20$ was adopted for input variables in the fitting model. All analyses were performed separately for each gender; $5 \%$ significance level was adopted.

\section{RESULTS}

The sample was composed of 1,984 adolescents, $55.9 \%$ of which were female. Among these, 748 (37.7\%) only reported using active transportation to school in a typical week: $16.2 \%$ boys and $21.5 \%$ girls. Tables 1 and 2 show the data stratified by gender.

Table 3 shows the association between sociodemographic factors and social support for active transportation to school (ATS) among boys. Regarding sociodemographic factors, we found no association between sociodemographic variables and ATS. As for social support from parents, results obtained by the adjusted analysis show that boys whose parents encourage physical activity (PA) practice were less likely to use active transportation (sometimes: odds ratio $[\mathrm{OR}]=0.64 ; 95 \%$ CI $0.42-0.96$; always: $\mathrm{OR}=0.58$; 95\%CI $0.36-0.95$ ). Conversely, boys were more likely to use ATS when parents sometimes practice PA with them $(\mathrm{OR}=1.57$; 95\% CI 1.09-2.25), always offer transportation for the PA practice $(\mathrm{OR}=$ 1.56 ; $95 \% \mathrm{CI} 1.04-2.32)$, and always remark their good performance in the PA (OR = 1.73; $95 \%$ CI 1.08-2.76).

Regarding social support from friends, boys who reported having friends that always practice PA were more likely to use ATS (OR $=2.23$; 95\%CI 1.35-3.69). Boys who have friends that watch them performing PA were less likely to use ATS (sometimes: OR $=0.63$; 95\% CI 0.42 0.95; always: $\mathrm{OR}=0.45 ; 95 \% \mathrm{CI} 0.25-0.79$ ).

Table 4 shows the association between sociodemographic factors and social support for ATS for boys. Among the evaluated sociodemographic variables, only age was significant in the adjusted model - girls aged 16 years were more likely to use ATS (OR $=1.43$; 95\%CI

Table 1. Adolescents' age, parental schooling, and socioeconomic status (SES) according to gender ( $\mathrm{n}=1,984)$.

\begin{tabular}{|c|c|c|c|c|c|c|c|c|}
\hline & & \multicolumn{2}{|c|}{$\begin{array}{c}\text { Male } \\
(n=875 ; 44.1 \%)\end{array}$} & \multicolumn{2}{|c|}{$\begin{array}{c}\begin{array}{c}\text { Female } \\
(\mathrm{n}=1,109 \\
55.9 \%)\end{array} \\
\end{array}$} & \multirow[b]{2}{*}{$\mathbf{p}^{\mathrm{c}}$} & \multicolumn{2}{|c|}{ Total } \\
\hline & & $\mathbf{n}$ & $\%$ & $\mathbf{n}$ & $\%$ & & $\mathbf{n}$ & $\%$ \\
\hline \multirow{3}{*}{ Age } & 15 years & 261 & 41.8 & 363 & 58.2 & 0.124 & 624 & \multirow{3}{*}{100} \\
\hline & 16 years & 317 & 44.3 & 399 & 55.7 & & 716 & \\
\hline & 17 years & 297 & 46.1 & 347 & 53.9 & & 644 & \\
\hline \multirow{3}{*}{ Father's schooling } & Elementary school & $238^{a}$ & 38.1 & $387^{\mathrm{a}}$ & 61.9 & 0.003 & 625 & \multirow{3}{*}{100} \\
\hline & High school & $405^{b}$ & 47.2 & $453^{\mathrm{b}}$ & 52.8 & & 858 & \\
\hline & College & $232^{\mathrm{b}}$ & 46.3 & $269^{b}$ & 53.7 & & 501 & \\
\hline \multirow{3}{*}{ Mother's schooling } & Elementary school & $245^{\mathrm{a}}$ & 39.0 & $384^{\mathrm{a}}$ & 61.0 & 0.006 & 629 & \multirow{3}{*}{100} \\
\hline & High school & $398^{\mathrm{b}}$ & 46.3 & $461^{\mathrm{b}}$ & 53.7 & & 859 & \\
\hline & College & $232^{\mathrm{b}}$ & 46.8 & $264^{\mathrm{b}}$ & 53.2 & & 496 & \\
\hline \multirow{3}{*}{ SES } & Lower & 140 & 40.0 & 210 & 60.0 & 0.005 & 350 & \multirow{3}{*}{100} \\
\hline & Middle & $538^{a, b}$ & 43.4 & $702^{a, b}$ & 56.6 & & 1,240 & \\
\hline & Higher & 197 & 50.0 & 197 & 50.0 & & 394 & \\
\hline
\end{tabular}

a,b Significantly differ from each other; post hoc of Bonferroni.

${ }^{\mathrm{c}}$ Chi-square test. 
1.06-1.92). Regarding variables related to social support (SS) for PA, only those referring to SS from friends remained associated in the adjusted model - girls who have friends who sometimes practice PA together with them are more likely to use ATS than those who never get this type of support ( $\mathrm{OR}=1.48 ; 95 \% \mathrm{CI} 1.04-2.10)$. Conversely, girls who have friends who sometimes invite them to practice PA were less likely to use ATS (OR = 0.65; 95\% CI 0.46-0.93).

Table 2. Prevalence of social support from parents and friends among adolescents according to gender $(\mathrm{n}=1,984)$.

\begin{tabular}{ccccccc}
$\begin{array}{c}\text { Male } \\
(\mathrm{n}=\mathbf{8 7 5} ; \mathbf{4 4 . 1} \%)\end{array}$ & $\begin{array}{c}\text { Female } \\
(\mathrm{n=1,109;} \\
55.9 \%)\end{array}$ & & \multicolumn{2}{c}{ Total } \\
\hline $\mathrm{n}$ & $\%$ & $\mathrm{n}$ & $\%$ & $\mathrm{p}^{\mathrm{c}}$ & $\mathrm{n}$ & $\%$ \\
\hline
\end{tabular}

\begin{tabular}{lcccccccc} 
Social support from parents & & & & & & & \\
\hline \multirow{2}{*}{ Encourage } & Never & 170 & 41.5 & 240 & 58.5 & 0.113 & 410 & \\
& Sometimes & 473 & 43.9 & 604 & 56.1 & 1,077 & 100 \\
& Always & 232 & 46.7 & 265 & 53.3 & 497 & \\
\hline \multirow{2}{*}{ Practice } & Never & 322 & 41.4 & 455 & 58.6 & 0.027 & 777 & \\
& Sometimes & 434 & 45.4 & 530 & 55.0 & & 964 & 100 \\
& Always & 119 & 49.0 & 124 & 51.0 & & 243 & \\
\hline \multirow{2}{*}{$\begin{array}{l}\text { Give a ride } \\
\text { transportation) }\end{array}$} & Never & 425 & 44.0 & 541 & 56.0 & 0.513 & 966 & \\
& Sometimes & 262 & 42.6 & 353 & 57.4 & & 615 & 100 \\
& Always & 188 & 46.7 & 215 & 53.3 & & 403 & \\
\hline \multirow{2}{*}{ Watch } & Never & 370 & 42.5 & 500 & 57.5 & 0.166 & 870 & \\
& Sometimes & 365 & 44.8 & 450 & 55.2 & & 815 & 100 \\
& Always & 140 & 46.8 & 159 & 53.2 & & 299 & \\
\hline \multirow{2}{*}{ Remark } & Never & 324 & 41.9 & 449 & 58.1 & 0.621 & 773 & \\
& Sometimes & 341 & 47.6 & 375 & 52.4 & & 716 & 100 \\
\hline & Always & 210 & 42.4 & 285 & 57.6 & & 495 & \\
\hline
\end{tabular}

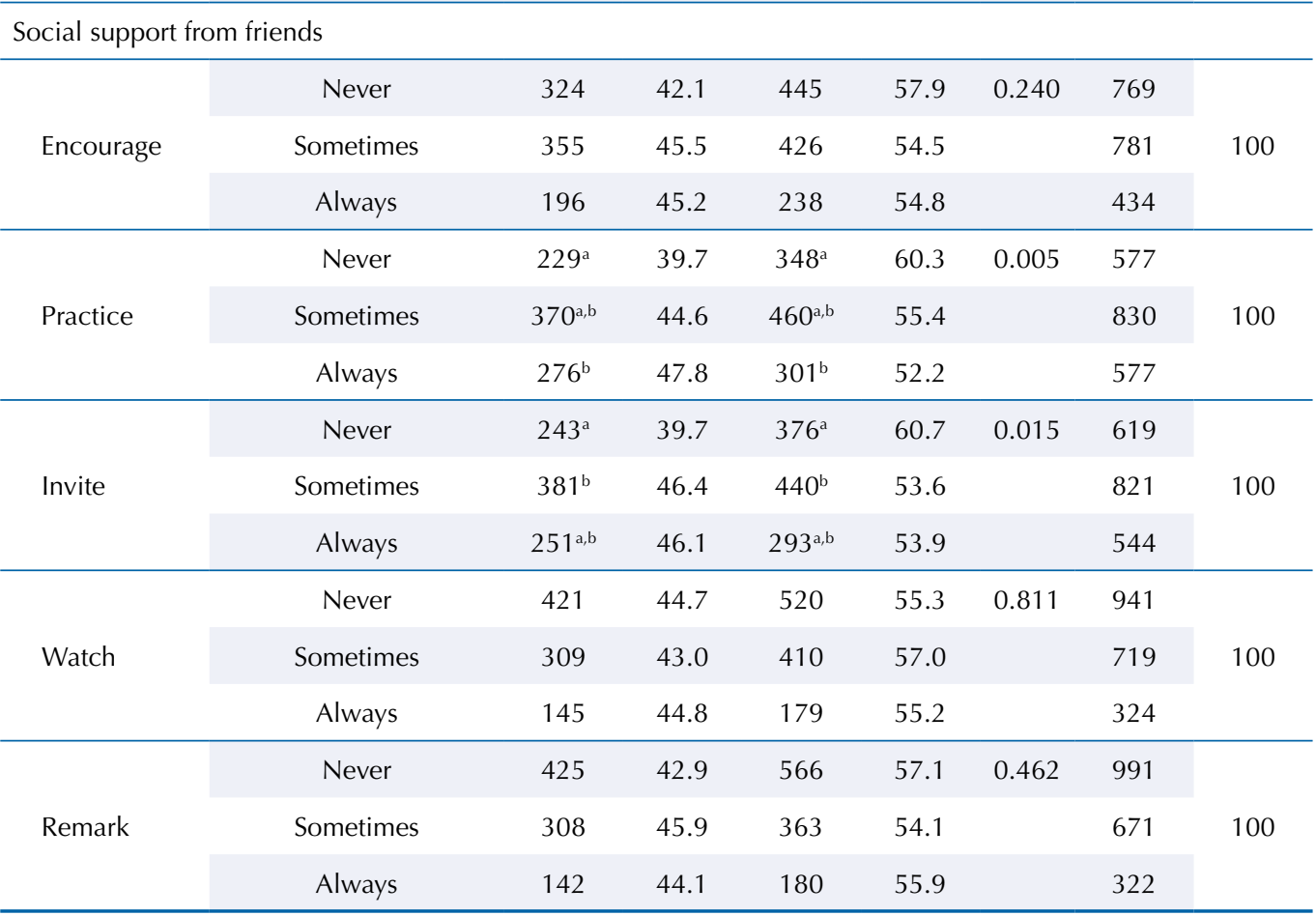

a,b Significantly differ from each other post hoc of Bonferroni

${ }^{a}$ Chi-square test. 
Table 3. Crude and adjusted association of sociodemographic factors and social support from parents and friends for active transportation to school among boys $(\mathrm{n}=875)$.

\begin{tabular}{|c|c|c|c|c|c|c|c|c|c|}
\hline \multirow{2}{*}{\multicolumn{2}{|c|}{ Sociodemographic factors }} & \multicolumn{5}{|c|}{ Crude } & \multicolumn{3}{|c|}{ Adjusted } \\
\hline & & $\mathrm{n}$ & $\%$ & OR & $95 \% \mathrm{Cl}$ & p & OR & $95 \% \mathrm{Cl}$ & p \\
\hline \multirow{3}{*}{ Age } & 15 years & 98 & 44.3 & 1.00 & & & & & \\
\hline & 16 years & 129 & 43.1 & 1.14 & $0.81-1.60$ & 0.441 & & & \\
\hline & 17 years & 95 & 41.7 & 0.78 & $0.55-1.11$ & 0.169 & & & \\
\hline \multirow{3}{*}{$\begin{array}{l}\text { Father's } \\
\text { schooling }\end{array}$} & Elementary school & 151 & 38.8 & 1 & & & & & \\
\hline & High school & 251 & 47.8 & 1.13 & $0.79-1.61$ & 0.508 & & & \\
\hline & College & 151 & 46.9 & 1.02 & $0.67-1.55$ & 0.924 & & & \\
\hline \multirow{3}{*}{$\begin{array}{l}\text { Mother's } \\
\text { schooling }\end{array}$} & Elementary school & 96 & 37.6 & 1.00 & & & & & \\
\hline & High school & 146 & 45.8 & 0.87 & $0.61-1.23$ & 0.422 & & & \\
\hline & College & 80 & 46.0 & 0.81 & $0.54-1.23$ & 0.321 & & & \\
\hline \multirow{3}{*}{ SES } & Lower & 49 & 37.4 & 1.00 & & & & & \\
\hline & Middle & 192 & 42.0 & 1.03 & $0.70-1.52$ & 0.880 & & & \\
\hline & Higher & 81 & 50.6 & 1.30 & $0.83-2.03$ & 0.256 & & & \\
\hline \multicolumn{2}{|c|}{ Social support from parents } & $\mathbf{n}$ & $\%$ & OR & $95 \% \mathrm{Cl}$ & p & OR & $95 \% \mathrm{Cl}$ & p \\
\hline \multirow{3}{*}{ Encourage } & Never & 67 & 38.7 & 1.00 & & & 1.00 & & \\
\hline & Sometimes & 169 & 43.4 & 0.67 & $0.45-1.00$ & 0.051 & 0.64 & $0.42-0.96$ & 0.033 \\
\hline & Always & 86 & 46.2 & 0.60 & $0.37-0.98$ & 0.039 & 0.58 & $0.36-0.95$ & 0.030 \\
\hline \multirow{3}{*}{ Practice } & Never & 99 & 35.7 & 1.00 & & & 1.00 & & \\
\hline & Sometimes & 178 & 46.8 & 1.49 & $1.05-2.10$ & 0.026 & 1.57 & $1.09-2.25$ & 0.015 \\
\hline & Always & 45 & 49.5 & 1.19 & $0.70-2.03$ & 0.522 & 1.23 & $0.72-2.09$ & 0.443 \\
\hline \multirow{3}{*}{$\begin{array}{l}\text { Give a ride } \\
\text { (or provide } \\
\text { transportation) }\end{array}$} & Never & 140 & 38.5 & 1.00 & & & 1.00 & & \\
\hline & Sometimes & 95 & 42.4 & 1.06 & $0.75-1.51$ & 0.735 & 1.03 & $0.72-1.48$ & 0.869 \\
\hline & Always & 87 & 54.4 & 1.64 & $1.11-2.41$ & 0.013 & 1.56 & $1.04-2.32$ & 0.030 \\
\hline \multirow{3}{*}{ Watch } & Never & 130 & 40.5 & 1.00 & & & & & \\
\hline & Sometimes & 139 & 43.6 & 0.83 & $0.57-1.21$ & 0.322 & & & \\
\hline & Always & 53 & 49.1 & 0.74 & $0.44-1.23$ & 0.247 & & & \\
\hline \multirow{3}{*}{ Remark } & Never & 99 & 36.4 & 1.00 & & & 1.00 & & \\
\hline & Sometimes & 134 & 47.5 & 1.55 & $1.04-2.32$ & 0.031 & 1.39 & $0.93-2.05$ & 0.105 \\
\hline & Always & 89 & 45.9 & 1.72 & $1.09-2.73$ & 0.020 & 1.73 & $1.08-2.76$ & 0.023 \\
\hline \multicolumn{2}{|c|}{ Social support from friends } & $\mathbf{n}$ & $\%$ & OR & $95 \% \mathrm{Cl}$ & p & OR & $95 \% \mathrm{Cl}$ & p \\
\hline \multirow{3}{*}{ Encourage } & Never & 117 & 40.1 & 1.00 & & & & & \\
\hline & Sometimes & 137 & 46.3 & 0.93 & $0.64-1.34$ & 0.687 & & & \\
\hline & Always & 68 & 42.5 & 0.65 & $0.40-1.06$ & 0.082 & & & \\
\hline \multirow{3}{*}{ Practice } & Never & 70 & 34.8 & 1.00 & & & 1.00 & & \\
\hline & Sometimes & 140 & 43.5 & 1.54 & $1.00-2.39$ & 0.050 & 1.50 & $0.97-2.30$ & 0.066 \\
\hline & Always & 112 & 49.8 & 1.97 & $1.16-3.33$ & 0.011 & 2.23 & $1.35-3.69$ & 0.002 \\
\hline \multirow{3}{*}{ Invite } & Never & 81 & 33.8 & 1.00 & & & & & \\
\hline & Sometimes & 138 & 46.8 & 1.03 & $0.65-1.58$ & 0.954 & & & \\
\hline & Always & 103 & 48.4 & 1.40 & $0.82-2.38$ & 0.222 & & & \\
\hline \multirow{3}{*}{ Watch } & Never & 157 & 43.7 & 1.00 & & & 1.00 & & \\
\hline & Sometimes & 115 & 42.4 & 0.70 & $0.47-1.04$ & 0.076 & 0.63 & $0.42-0.95$ & 0.026 \\
\hline & Always & 50 & 42.4 & 0.53 & $0.30-0.93$ & 0.026 & 0.45 & $0.25-0.79$ & 0.006 \\
\hline \multirow{3}{*}{ Remark } & Never & 146 & 39.7 & 1.00 & & & & & \\
\hline & Sometimes & 122 & 46.7 & 1.30 & $0.90-1.88$ & 0.165 & & & \\
\hline & Always & 54 & 45.4 & 1.31 & $0.78-2.19$ & 0.304 & & & \\
\hline
\end{tabular}

OR: odds ratio; $95 \% \mathrm{Cl}$ : $95 \%$ confidence interval

Note: Only the variables presenting $\mathrm{p}$ values $\leq 0.20$ in the crude analysis remained in the adjusted analysis. 
Table 4. Crude and adjusted association of sociodemographic factors and social support from parents and friends for active transportation to school among girls $(n=1,109)$.

\begin{tabular}{|c|c|c|c|c|c|c|c|c|c|}
\hline \multirow{2}{*}{\multicolumn{2}{|c|}{ Sociodemographic factors }} & \multicolumn{5}{|c|}{ Crude } & \multicolumn{3}{|c|}{ Adjusted } \\
\hline & & \multirow{2}{*}{$\begin{array}{c}\mathbf{n} \\
123\end{array}$} & \multirow{2}{*}{$\begin{array}{c}\% \\
55.7\end{array}$} & \multirow{2}{*}{$\begin{array}{c}\text { OR } \\
1.00\end{array}$} & \multirow[t]{2}{*}{$95 \% \mathrm{Cl}$} & \multirow[t]{2}{*}{$\mathbf{p}$} & \multirow{2}{*}{$\begin{array}{c}\text { OR } \\
1.00\end{array}$} & \multirow[t]{2}{*}{$95 \% \mathrm{Cl}$} & \multirow[t]{2}{*}{$\mathbf{p}$} \\
\hline \multirow{3}{*}{ Age } & 15 years & & & & & & & & \\
\hline & 16 years & 170 & 56.9 & 1.45 & $1.08-1.95$ & 0.014 & 1.43 & $1.06-1.92$ & 0.018 \\
\hline & 17 years & 133 & 58.3 & 1.21 & $0.89-1.65$ & 0.218 & 1.17 & $0.86-1.60$ & 0.317 \\
\hline \multirow{3}{*}{$\begin{array}{l}\text { Father's } \\
\text { schooling }\end{array}$} & Elementary school & 238 & 61.2 & 1.00 & & & & & \\
\hline & High school & 274 & 52.2 & 1.13 & $0.84-1.53$ & 0.418 & & & \\
\hline & College & 171 & 53.1 & 1.03 & $0.71-1.48$ & 0.854 & & & \\
\hline \multirow{3}{*}{$\begin{array}{l}\text { Mother's } \\
\text { schooling }\end{array}$} & Elementary school & 159 & 62.4 & 1.00 & & & & & \\
\hline & High school & 173 & 54.2 & 0.82 & $0.60-1.10$ & 0.182 & & & \\
\hline & College & 94 & 54.0 & 0.77 & $0.54-1.11$ & 0.156 & & & \\
\hline \multirow{3}{*}{ SES } & Lower & 82 & 62.6 & 1.00 & & & & & \\
\hline & Middle & 265 & 58.0 & 0.95 & $0.70-1.30$ & 0.734 & & & \\
\hline & Higher & 79 & 49.4 & 1.05 & $0.70-1.56$ & 0.828 & & & \\
\hline \multicolumn{2}{|c|}{ Social support from parents } & $\mathbf{n}$ & $\%$ & OR & $95 \% \mathrm{Cl}$ & $\mathbf{p}$ & OR & $95 \% \mathrm{Cl}$ & $\mathbf{p}$ \\
\hline \multirow{3}{*}{ Encourage } & Never & 106 & 61.3 & 1.00 & & & & & \\
\hline & Sometimes & 220 & 56.6 & 0.70 & $0.50-0.98$ & 0.040 & & & \\
\hline & Always & 100 & 53.8 & 0.80 & $0.52-1.23$ & 0.314 & & & \\
\hline \multirow{3}{*}{ Practice } & Never & 178 & 64.3 & 1.00 & & & & & \\
\hline & Sometimes & 202 & 53.2 & 1.04 & $0.77-1.40$ & 0.809 & & & \\
\hline & Always & 46 & 50.5 & 1.03 & $0.63-1.66$ & 0.921 & & & \\
\hline \multirow{3}{*}{$\begin{array}{l}\text { Give a ride } \\
\text { (or provide } \\
\text { transportation) }\end{array}$} & Never & 224 & 61.5 & 1.00 & & & & & \\
\hline & Sometimes & 129 & 57.6 & 0.79 & $0.58-1.07$ & 0.124 & & & \\
\hline & Always & 73 & 45.6 & 0.71 & $0.48-1.04$ & 0.076 & & & \\
\hline \multirow{3}{*}{ Watch } & Never & 191 & 59.5 & 1.00 & & & & & \\
\hline & Sometimes & 180 & 56.4 & 1.20 & $0.87-1.66$ & 0.258 & & & \\
\hline & Always & 55 & 50.9 & 0.98 & $0.62-1.54$ & 0.919 & & & \\
\hline \multirow{3}{*}{ Remark } & Never & 173 & 63.6 & 1.00 & & & & & \\
\hline & Sometimes & 148 & 52.5 & 1.18 & $0.84-1.66$ & 0.340 & & & \\
\hline & Always & 105 & 54.1 & 1.08 & $0.72-1.61$ & 0.722 & & & \\
\hline Social support $f$ & friends & $\mathbf{n}$ & $\%$ & OR & $95 \% \mathrm{Cl}$ & $\mathbf{p}$ & OR & $95 \% \mathrm{Cl}$ & p \\
\hline & Never & 175 & 59.9 & 1.00 & & & & & \\
\hline Encourage & Sometimes & 159 & 53.7 & 0.98 & $0.70-1.37$ & 0.908 & & & \\
\hline & Always & 92 & 57.5 & 1.09 & $0.72-1.66$ & 0.683 & & & \\
\hline & Never & 131 & 65.2 & 1.00 & & & 1.00 & & \\
\hline Practice & Sometimes & 182 & 56.5 & 1.39 & $0.96-2.01$ & 0.084 & 1.48 & $1.04-2.10$ & 0.030 \\
\hline & Always & 113 & 50.2 & 1.24 & $0.79-1.95$ & 0.356 & 1.35 & $0.88-2.07$ & 0.176 \\
\hline & Never & 159 & 66.3 & 1.00 & & & 1.00 & & \\
\hline Invite & Sometimes & 157 & 53.2 & 0.62 & $0.43-0.91$ & 0.013 & 0.65 & $0.46-0.93$ & 0.018 \\
\hline & Always & 110 & 51.2 & 0.71 & $0.45-1.14$ & 0.160 & 0.73 & $0.48-1.11$ & 0.144 \\
\hline & Never & 202 & 56.3 & 1.00 & & & & & \\
\hline Watch & Sometimes & 156 & 57.6 & 1.00 & $0.72-1.40$ & 0.990 & & & \\
\hline & Always & 68 & 57.6 & 1.02 & $0.64-1.63$ & 0.942 & & & \\
\hline & Never & 222 & 60.3 & 1.00 & & & & & \\
\hline Remark & Sometimes & 139 & 53.3 & 1.05 & $0.75-1.48$ & 0.777 & & & \\
\hline & Always & 65 & 54.6 & 0.90 & $0.56-1.43$ & 0.649 & & & \\
\hline
\end{tabular}

OR: odds ratio; $95 \% \mathrm{Cl}$ : 95\% confidence interval

Note: Only the variables presenting $\mathrm{p}$ values $\leq 0.20$ in the crude analysis remained in the adjusted analysis. 


\section{DISCUSSION}

Adolescence is a critical moment for establishing physical activity (PA) habits; yet, the literature has depicted this life period with a gradual engagement decline across the various domains of $\mathrm{PA}^{16,17}$. Thus, our study targeted adolescents aged from 15 to 17 years. Active transportation to school (ATS) is an effective way of incorporating PA into daily activities and increasing adolescents' overall PA levels ${ }^{1,2}$ - especially during transition from late adolescence to young adulthood, a critical period for decreasing PA levels ${ }^{16-18}$. Mandic et al. ${ }^{19}$, point that adolescents' decision in adopting ATS may be influenced by several factors of personal, social, or environmental nature. Our research aimed to verify how sociodemographic factors (personal) and social support for PA practice (social) are associated with the use of ATS among adolescents enrolled in public schools in a large capital of Southern Brazil.

Regarding the prevalence of ATS in this research, $37 \%$ of the adolescents reported practicing this behavior. A recent systematic review on ATS among Brazilian adolescents identified a great variability in prevalence, ranging from $34.3 \%$ to $75.7 \%$, depending on the evaluated study $^{2}$. Most studies covered by this review reported a higher prevalence than that found in our research. However, only two of the studies included in this review involving national sampling presented data from adolescents from Curitiba ${ }^{2}$.

Regarding gender, we found that $21.5 \%$ of ATS is used by girls. Other studies ${ }^{1-4}$ also found a higher prevalence of active transportation among girls, but they are not unanimous, so these results should be interpreted with caution. Girls' PA levels are lower than their peers in the context of leisure ${ }^{18}$, so transportation may be a way to increase girls' PA. However, further research is required to investigate the prevalence of ATS considering gender. We should also consider that our research deals with ATS to and from school, while others deal only with one-way ATS. Validating instruments on active transportation for specific populations could help standardizing measures.

Studies have considered sociodemographic factors as possible influencers of ATS among adolescents ${ }^{19}$. Our results show association between these factors and ATS use only among girls, specially at the age of 16 - which may be related to the fact that older girls are more autonomous than younger girls. Further investigations may address the possibility of maximizing girls' PA levels at the transport domain. Some studies show that older adolescents (both boys and girls) often use passive transport modes, such as car and motorcycle, even to nearby destinations ${ }^{1,20}$. These results suggest a need for greater efforts to understand factors that may influence the use of ATS among older adolescents, aiming to increase the use of this mode of commuting to school and other destination $\mathbf{s}^{1,20}$.

Conversely, this study found no association between ATS and sociodemographic factors such as parental schooling and socioeconomic status, deemed as important predictors of the different PA contexts (transportation and leisure) $)^{16,20,21}$. What motivates these results is still unclear, but a possibility is that higher parental schooling and socioeconomic status encounter different barriers to ATS, such as schedules or perception of safety ${ }^{1-3}$, regardless of the support for PA practice in other contexts.

Social support is associated with adolescents' increased engagement in the different domains of $\mathrm{PA}^{20}$. The literature suggests that adolescents (boys and girls) report higher PA levels when their parents and friends provide greater support. ${ }^{13}$ Our study found that ATS was favored among boys who have parents that practice PA together with them, provide transportation for PA, and remark their good performance on it. We also observed that boys who have friends that practice PA with them are twice as likely to use ATS.

As for girls, having friends who practice PA with them was associated with ATS. Some studies state that the lack of company is the main barrier for girls to practice PA in leisure time $^{22}$. This suggests that having friends who invite them may increase the odds of girls being active in the different contexts of PA (leisure and transportation). Our study corroborates this line by investigating the context of transportation. 
Interestingly, the use of ATS was less likely among boys with encouraging parents and friends who watch their PA practice, and girls with friends who invite them to practice PA. This study does not explain what motivates such associations, but it reinforces the need for a more detailed research on how each social support characteristic can impact different types of PA practice among adolescents, possibly integrating quantitative and qualitative methods of research. Both genders presented associations related to social support from friends, differently from social support from parents, which was associated only in boys. This may be explained by a greater influence of parents for male children when it comes to practicing PA, enabling this finding to be a discussion topic for future studies investigating actions for gender equity.

When interpreting this study results, we should consider some limitations. First, due to the cross-sectional design, no causal relationship can be drawn from the results. Second, a self-reported questionnaire may lead participants to overestimate or underestimate the use of the transport modes at issue. The lack of knowledge regarding distance is a fundamental limitation. Further studies should consider including objective measures (GPS, for example) and subjective measures of transport behavior. Our study sample is formed only by students from public schools, which precludes the extrapolation of results to higher classes. However, the representative sample and statistical analyses ensure data interpretation for large populations of public schools - a key point in the field of interventions related to public health and prevention. This study also corroborates the investigation of sociodemographic and psychosocial factors for ATS among a representative sample of adolescents. We did not investigate the significant association between ATS and body mass index (BMI) and recommended levels of physical activity; however, we suggest that further studies evaluate this relationship for a greater clarification on ATS.

\section{CONCLUSION}

The results show a $37.7 \%$ prevalence of active transportation to school (ATS): $16.2 \%$ among boys and $21.5 \%$ among girls. We found no association between sociodemographic factors and ATS, except age for girls. Social support for physical activity (PA) practice, from both parents and friends, was associated with ATS. For boys, ATS was associated with having parents who: practice PA together with them, provide transportation for PA, and remark their good performance on it; as well as having friends who practice PA together with them. For girls, ATS was solely associated with age and having friends who practice PA together with them.

\section{REFERENCES}

1. Verhoeven H, Simons D, Van Dyck D, Van Cauwenberg J, Clarys P, De Bourdeaudhuij I, et al. Psychosocial and environmental correlates of walking, cycling, public transport and passive transport to various destinations in Flemish older adolescents. PLoS One. 2016;11(1):e0147128. https://doi.org/10.1371/journal.pone.0147128

2. Ferrari GLM, Victo ER, Ferrari TK, Solé D. Active transportation to school for children and adolescents from Brazil: a systematic review. Rev Bras Cineantropom Desempenho Hum. 2018;20(4):406-14. https://doi.org/10.5007/1980-0037.2018v20n4p406

3. Ramírez-Vélez R, García-Hermoso A, Agostinis-Sobrinho C, Mota J, Santos R, Correa-Bautista JE, et al. Cycling to school and body composition, physical fitness, and metabolic syndrome in children and adolescents. J. Pediatr. 2017; 188:57-63. https://doi.org/10.1016/j.jpeds.2017.05.065

4. Pizarro AN, Schipperijn J, Andersem HB, Ribeiro JC, Mota J, Santos MP, et al. Active commuting to school in Portuguese adolescents: using PALMS to detect trips. J Transp Health. 2016;3(3):297-304. https://doi.org/10.1016/j.jth.2016.02.004

5. Bauman AE, Reis RS, Sallis JF, Wells JC, Loos RJF, Martin BW. Correlates of physical activity: why are some people physically active and others not? Lancet. 2012;380(9838):258-71. https://doi.org/10.1016/S0140-6736(12)60735-1 
6. Sallis JF, Bull F, Guthold R, Heath GW, Inoue S, Kelly P, et al. Progress in physical activity over the Olympic quadrennium. Lancet. 2016;388(10051):1325-36. https://doi.org/10.1016/S0140-6736(16)30581-5

7. Kienteka M. A implantação de ciclofaixas e o padrão de uso de bicicleta em Curitiba-PR [tese]. Curitiba, PR: Universidade Federal do Paraná; 2017.

8. Perry CK, Garside H, Morones S, Hayman LL. Physical activity interventions for adolescents: an ecological perspective. J Prim Prev. 2012;33(2-3);111-35. https://doi.org/10.1007/s10935-012-0270-3

9. Fermino RC, Rech CR, Hino AAF, Rodriguez Añez CR, Reis RS. Physical activity and associated factors in high school adolescents in Southern Brazil. Rev Saude Publica. 2010;44(6):986-95. https://doi.org/10.1590/s0034-89102010000600002

10. Associação Brasileira de Empresas de Pesquisa. Critério de Classificação Econômica. São Paulo ABEP; 2018.

11. Farias Júnior JC, Mendonça G, Florindo AA, Barros MVG. Reliability and validity of a physical activity social support assessment scale in adolescents - ASAFA Scale. Rev Bras Epidemiol 2014;17(2):355-70. https://doi.org/10.1590/1809-4503201400020006ENG

12. Piola TS, Bacil EDA, Watanabe PI, Camargo EM, Fermino RC, Campos W. Sexual maturation, social support and physical activity in adolescents. Cuad Psicol Deporte. 2019;19(3):CPD3. https://doi.org/10.6018/cpd.347821

13. Mendonça, Cheng LA, Melo EN, Farias Junior JC. Physical activity and social support in adolescents: a systematic review. Health Educ Res. 2014;29(5):822-39. https://doi.org/10.1093/her/cyu017

14. Wong BYM, Faulkner G, Buliung R. GIS measured environmental correlates of active school transport: a systematic review of 14 studies. Int J Behav Nutr Phys Act. 2011;8:39. https://doi.org/10.1186/1479-5868-8-39

15. Becker LA, Fermino RC, Lima AV, Rech CR, Añez CR, Reis RS. Perceived barriers for active commuting to school among adolescents from Curitiba, Brasil. Rev Bras Ativ Fis Saude. 2017;22(1):24-34. https://doi.org/10.12820/rbafs.v.22n1p24-34

16. Dumith SC, Gigante DP, Domingues MR, Hallal PC, Menezes AMB, Kohl HW 3rd. A longitudinal evaluation of physical activity in Brazilian adolescents: tracking, change and predictors. Pediatr Exerc Sci. 2012;24(8):58-71. https://doi.org/10.1123/pes.24.1.58

17. Wiium N, Breivik K, Wold B. Growth trajectories of health behaviors from adolescence through young adulthood. Int J Environ Res Public Health. 2015;12(11):13711-29. https://doi.org/10.3390/ijerph121113711

18. Silva AAP, Camargo EM, Silva AT, Silva JSB, Hino AAF, Reis RS. Characterization of physical activities performed by adolescents from Curitiba, Brazil. Rev Bras Med Esporte. 2019;25(3):211 5. https://doi.org/10.1590/1517-869220192503188171

19. Mandic S, Leon de la Barra S, García Bengoechea E, Stevens E, Flaherty C, Moore A, et al. Personal, social and environmental correlates of active transport to school among adolescents in Otago, New Zealand. J Sci Med Sport. 2015;18(4):432-7. https://doi.org/10.1016/j.jsams.2014.06.012

20. Silva KS, Pizarro AN, Garcia LMT, Mota J, Santos MP. Which social support and psychological factors are associated to active commuting to school? Prev Med. 2014;63:20-3. https://doi.org/10.1016/j.ypmed.2014.02.019

21. Farias Junior JC, Reis RS, Hallal PC. Physical activity, psychosocial and perceived environmental factors in adolescents from Northeast Brasil. Cad Saude Publica. 2014;30(5):941-51. https://doi.org/10.1590/0102-311X00010813

22. Rech CR, Camargo EM, Araujo PAB, Loch MR, Reis RS. Perceived barriers to leisure-time physical activity in the Brazilian population. Rev Bras Med Esporte. 2018;24(4):303-9. https://doi.org/10.1590/1517-869220182404175052

Funding: This study was funded by the Coordenação de Aperfeiçoamento de Pessoal de Nível Superior (CAPES), Brazil, funding code 001. It was supported by grants: FCT: SFRH/BSAB/142983/2018 and UID/DTP/00617/2019, as well as the Santander Universities Scholarship 2018.

Author's Contribution: EMC: Responsible for all aspects of the study. MPS: Writing of the manuscript. JM: Critical review of the manuscript. WC: Critical review of the manuscript.

Conflict of Interest: The authors declare no conflict of interest. 